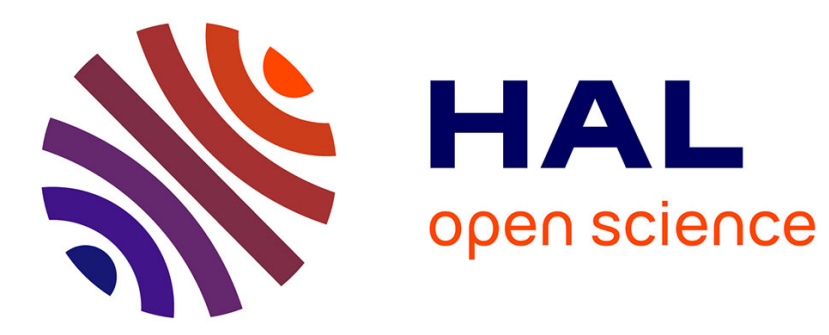

\title{
Chromatin mobility upon DNA damage: state of the art and remaining questions
}

Christophe Zimmer, Emmanuelle Fabre

\section{To cite this version:}

Christophe Zimmer, Emmanuelle Fabre. Chromatin mobility upon DNA damage: state of the art and remaining questions. Current Genetics, 2019, 65 (1), pp.1-9. 10.1007/s00294-018-0852-6 . pasteur02074352

\section{HAL Id: pasteur-02074352}

\section{https://hal-pasteur.archives-ouvertes.fr/pasteur-02074352}

Submitted on 6 Mar 2020

HAL is a multi-disciplinary open access archive for the deposit and dissemination of scientific research documents, whether they are published or not. The documents may come from teaching and research institutions in France or abroad, or from public or private research centers.
L'archive ouverte pluridisciplinaire HAL, est destinée au dépôt et à la diffusion de documents scientifiques de niveau recherche, publiés ou non, émanant des établissements d'enseignement et de recherche français ou étrangers, des laboratoires publics ou privés. 
Chromatin mobility upon DNA damage: state of the art and remaining questions.

Christophe Zimmer ${ }^{1,2}$ and Emmanuelle Fabre ${ }^{3,4}$

${ }^{1}$ Institut Pasteur, Unité Imagerie et Modélisation, 25 rue du Docteur Roux, 75015 Paris, France

${ }^{2}$ UMR 3691, CNRS; C3BI, USR 3756, IP CNRS

${ }^{3}$ Equipe Biologie et Dynamique des Chromosomes, Institut Universitaire d'Hématologie, Hôpital St. Louis, Paris, France

${ }^{4}$ CNRS UMR 7212, INSERM U944, IUH, Université Paris Diderot Sorbonne Paris Cité, Paris, France

\begin{abstract}
Chromosome organization and chromatin mobility are central to DNA metabolism. In particular, it has been recently shown by several labs that Double Strand Breaks (DSBs) in yeast induce a change in chromatin mobility at the site of the damage. Intriguingly, DSB also induces a global mobility of the genome, at others, potentially undamaged positions. How mobility is regulated and what are the functional outcomes of these global changes in chromatin dynamics are however not yet fully understood. We present the current state of knowledge in light of recent literature and discuss some perspectives opened by these discoveries towards genome stability.
\end{abstract}

\title{
Keywords
}

Chromatin, Double strand breaks, mobility, polymer physics, yeast 


\section{Assessing chromatin mobility}

Chromatin of any living organism is under constant motion and is probably essential for most, if not all, DNA transactions including replication, transcription and repair. To address functional outcomes, defining how a moving particle is displaced is important, since it permits to apprehend the way this particle explores its surrounding space and the time it might take to create contacts with functional molecular partners.

Mean Square Displacement (MSD) is the common measure to define the space explored by a randomly diffusing particle ((Albert et al. 2012; Spichal and Fabre 2017) for recent reviews). When a particle diffuses freely, MSD curve increases proportionally with time. When particle free diffusion happens in a constrained space, the MSD curve shows a plateau defining a radius of confinement $\left(R_{c}\right)$ for the moving particle. An increased $R_{c}$, visible at long time points, is often interpreted as the result of a faster diffusion or a less constrained diffusion although only the slope at the origin of the curve, at short time points, can define this. In yeast, initial time lapse particle tracking studies described motion of genomic loci as free diffusion in a restricted space (Marshall et al. 1997; Heun 2001). However, MSD of a moving particle scales as $\mathrm{Dt}^{\alpha}$. D values inform on the speed with which the particle moves and $\alpha$ values inform on the space explored by the particle: the smaller $\alpha$, the more often the particle will explore the same area. The motion is named subdiffusive when $\alpha$ values are below 1 . Several studies in yeast have now established that $\alpha$ ranges from $\sim 0.4$ to $\sim 0.76$ and the prefactor D lies between 1.2 and $210^{-3} \mathrm{\mu m}^{2} / \mathrm{sec}^{\alpha}$ (Cabal et al. 2006; Hajjoul et al. 2013; Backlund et al. 2014; Spichal et al. 2016; Hauer et al. 2017; Miné-Hattab et al. 2017; Herbert et al. 2017). Contrary to confined diffusion, particle subdiffusive motion reflects a constrained motion without boundaries. Subdiffusion can be explained by crowding, viscoelastic properties of the environment and/or behavior of a locus embedded within chromatin and retained by juxtaposing neighbors in a polymeric chromatin chain (Weber et al. 2010). The fact that subdiffusive motion of chromatin was observed in bacteria and mammalian cells, suggests a universal dynamic behavior of chromatin in non challenging conditions (Weber et al. 2010; Bronshtein et al. 2015).

\section{Factors involved in chromatin motion}

To follow chromatin motion, the most popular tool remains the tracking of bacterial repeats LacO or TetO inserted into the genome, although interesting alternatives are developed \{Belmont:2001tj\}; Bystricky:2015fj\}. When bound by their cognate repressor fused to a fluorescent protein, MSD can be calculated at different time scales. Different physiological parameters are known to affect chromatin motion in yeast. First, locus position in the nucleus is important; proximity to tethering 
structures like the nuclear envelope, as in mammals, will restrict movements (Heun 2001; Chubb et al. 2002; Hajjoul et al. 2013; Spichal et al. 2016). In particular, centromere tethering by a nuclear microtubule clearly restricts mobility of an undamaged locus located $8.8 \mathrm{~kb}$ from the centromere (Marshall et al. 1997; Verdaasdonk et al. 2013; Lawrimore et al. 2017). Induction of transcription through the centromere - which corresponds to a single nucleosome in budding S.cerevisiae yeast detaches it from kinetochore and leads to a dramatic increase in mobility. Furthermore, the use of nocodazole that inhibits microtubules polymerization leads to larger $R_{c}$ of a centromere proximal locus (Marshall et al. 1997; Verdaasdonk et al. 2013; Lawrimore et al. 2017). A second factor is ploidy; chromatin mobility can appear faster in haploids than diploid cells for certain loci - for example URA3, which is located in the middle of chromosome VR (Miné-Hattab et al. 2017), but not for others - like GAL1-GAL10, which is close to the chromosome II centromere (Backlund et al. 2014). Cell cycle is a third factor; chromatin motion is slower in S phase than in G1 in haploid cells (Heun 2001; Dion et al. 2012). Fourth, growth conditions, like the nature of the carbon sources are important to consider. Mobility is observed to be slower in galactose than glucose is some cases (Backlund et al. 2014). In addition to these factors, chromatin remodeling, ATP driven, complexes are involved in energy consuming motion. For instance, direct targeting of catalytically active Arp8, the ATPase subunit of the chromatin remodelling INO80 complex, drives enhanced mobility of undamaged chromatin, through a mechanism proposed to involve nucleosome eviction (Neumann et al. 2012). Surprisingly, in this study, only INO80 remodelling complex, and not other complexes such as SWI/SNF also able to remove nucleosomes, were shown to be able to drive chromatin motion, as visualized by lacO tracking of the fiber (Neumann et al. 2012). Of note, actin, a constitutive component of the INO80 complex is involved in chromatin motion as judged from the MSD analyses of different labeled genomic loci in the presence of latrunculin A, a drug that inhibits actin polymerization (Spichal et al. 2016). How cytoskeleton proteins, chromatin remodeling complexes or cell physiology regulate chromatin motion in these undamaged conditions remains to be fully understood.

\section{Polymer physics and chromatin motion}

Polymer theory is of great help to understand the mechanism underlying chromatin motion. Polymer chains can be defined by their contour length $C$ in $(\mathrm{nm})$ and their Kuhn Length $L_{k}$, which is twice the persistence length $L_{p}$. $L_{p}$ defines the bending rigidity of the chain. By definition, the orientation between the segments linking two monomers becomes uncoupled if the distance between these 
monomers is greater than $L_{P}{ }^{1}$. The Rouse model describes the dynamics of an ideal chain. This chain is composed of monomers connected by elastic segments subjected to springs oscillation. Each monomer, subjected to Brownian random motion, will define the conformational dynamics of the chain. This simple Rouse polymer dynamics predicts $\alpha$ scaling of $0.5 ; M S D(t) \approx L_{k}(D t)^{0.5}$. This is the $\alpha$ value found at several time scales for different chromosomal loci examined in yeast in a pioneering study (Hajjoul et al. 2013). Interestingly, chain properties like its compaction or its rigidity are expected to influence $\alpha$. The Rouse model predicts a reduction in chromatin mobility upon decondensation for times above the Rouse relaxation time $\tau_{\mathrm{R}}$ and an increased mobility for stiffer chromatin for times below the Rouse time (Steinhauser et al. 2009; Arbona et al. 2017; Herbert et al. 2017). Yet, in an exact Rouse model, $\alpha$ can gradually increase from 0.5 to 1 for increased time intervals, corresponding to the transition between a global subdiffusive behavior of the chain to a normal diffusion. There are different extensions of this model, for instance when the concentration of polymer chains that can topologically restrict chain motion is taken into account (i. $e$. entanglement described in the reptation model proposed by P-G. de Gennes for long chains, in this case an $\alpha=$ ? ?] ? ? ? is predicted) or when solvent properties are considered (i. e. hydrodynamic interactions in the Zimm Model).

\section{DNA Double strand breaks and chromatin motion}

Double strand breaks (DSBs) are a major threat for genomic integrity. Left unrepaired, cells will die, while repair between repeated sequences or mis-rejoining from different break sites could create chromosome aberrations with a potential risk for the cell. Studies in yeast have analyzed chromatin mobility upon DNA damage and found increased motion at the damaged locus and elsewhere in the potentially undamaged genome.

In these studies, DSBs were created either through the controlled induction of homing endonucleases HO or I-Scel (Miné-Hattab and Rothstein 2012; Dion et al. 2012; Miné-Hattab et al. 2017) or through the action of genotoxic chemotherapeutic agents, like antibiotics from the bleomycin glycopeptide family (including Zeocin or Phleomycin) or $\gamma$ Rays . In vitro, bleomycin was found to create specific concerted DNA strand scission trough oxygenation of the C-3'--C-4' bond of

\footnotetext{
${ }^{1}$ Stiffening is used with apparently contradictory meanings in the literature. In the case of an ideal polymer, oscillating springs connects monomers. On one hand, stiffness is defined by the tension exerted by the entire polymer when pulling on its ends. In this case, spring constant is expressed as $1 / L_{p}$. If $L_{p}$ is high, the polymer will moderately resist to elongation and will behave as a soft polymer (Verdaasdonk et al. 2013). On the other hand, stiffening is also used to directly define the bending rigidity of the polymer. If $L_{p}$ is high, the polymer will bend moderately and appear as stiff (Herbert et al. 2017). Therefore it is important to clearly address the meaning of stiffening when polymer properties are examined. In the present review we use the second definition.
} 
deoxyribose. Enzymes create "clean" breaks with 3'OH and 5'Phosphate DSB ends directly accessible to repair enzymes, while $\gamma$ irradiations can give rise to complex and heterogeneous breaks. Bleomycin creates 3'phosphoglycolate termini to be cleaned before repair. Once the DSB generated, at the G1/S transition, the cell cycle dependent checkpoint Cdc28 kinase controls a single stranded resection initiated at the DSB. Indeed, the MRX complex (Mre11, Rad50, Xrs2), in cooperation with Sae2, engages 5' to 3' resection and stimulates further resection by Exo1 and Sgs1-Dna2 (Figure 1). Single stranded DNA can extend up to several $\mathrm{kb}$ and is required for subsequent repair by homologous recombination. Single stranded DNA is bound by the Replication factor A complex RPA, Rad52 and bacterial recA orthologue, Rad51 (Tsukuda et al. 2005; Gibb et al. 2014) (Figure 1).

When DSBs are generated either enzymatically, or by genotoxic means, changes in chromatin mobility are observed. Locally, chromatin next to an HO induced DSB cut shows a faster motion, after a transient mobility reduction (Saad et al. 2014). In diploid cells, the Rc of a locus close to an IScel generated DSB increases at longer time points (Miné-Hattab and Rothstein 2012). Furthermore, tracking at short time scales, at $10 \mathrm{~ms}$, evidenced that damaged DNA was less mobile than undamaged DNA (Miné-Hattab et al. 2017). Interestingly this motion is Rad51 dependent, suggesting a role in the single-stranded bound chromatin structure in regulating DNA motion. Rad51 bound chromatin , proposed to act as a "needle to help search in the chromatin mesh", deserves super resolution microscopy observations (Miné-Hattab et al. 2017). Surprisingly, not only the broken chromosome shows increased mobility: several labs found that other chromosomes move as well, both in haploid and diploid yeast cells. How mobility is regulated and what is the purpose of this mobility is not well understood. However recent work summarized below, has generated some information about the genetic control, and about the different apparent behavior of chromatin at different scales (Miné-Hattab and Rothstein 2012; Dion et al. 2012; Seeber et al. 2013; Strecker et al. 2016; Hauer et al. 2017; Lawrimore et al. 2017; Miné-Hattab et al. 2017; Herbert et al. 2017).

\section{Potential factors driving global chromatin movement upon DNA damage}

Given our knowledge on chromatin motion, factors that may be at play in the global genome mobility can be: i) modification of internal components of the chromatin or of chromatin remodeling activities that can change the intrinsic physical properties of the chromatin upon damage; ii) factors, viewed as external forces, that can free constrained chromatin motion after DSBs; iii) modification of the viscoelastic properties of the nucleoplasm, a possibility not yet explored and not discussed here (figure 2).

\section{A. Intrinsic components of the chromatin fiber}


1. Checkpoint proteins One of the first steps in the DNA damage response (DDR) is the sensing of damaged DNA. Checkpoint kinase proteins and DSB repair proteins themselves are key for this DDR. As seen above, after a DSB, MRX complex can bind DSB ends. It then promotes the recruitment of kinase Tel1 (ATM in mammals) whose activation occurs prior to $5^{\prime}$ to $3^{\prime}$ DNA end resection. Single stranded DNA induces checkpoint signaling by Mec1 (ATR in mammals). Mec1 associates with Ddc2 (ATRIP in mammals), while the 9-1-1 complex (Ddc1 - Rad17- Mec3) clamp binds independently to single stranded RPA coated DNA (Finn et al. 2011). Direct Ddc1 and Ddc2 recruitment to chromatin was shown to promote checkpoint activation and local chromatin mobility, independently of a DSB (Bonilla et al. 2008; Seeber et al. 2013). Both Tel1 and Mec1 phosphorylate H2A on Ser129; local recruitment of Ddc1 and Ddc2 also induces H2A phosphorylation (Bonilla et al. 2008; Seeber et al. 2013). Domains of H2A phosphorylation can extend for $\sim 50 \mathrm{~kb}$ around the DSB, up to a third of the length of a chromosome arm (Shroff et al. 2004; Lee et al. 2014). Rad9 (53BP1 in mammals) is recruited through binding to phosphorylated H2A and constitutive methylated H3K79 (Grenon et al. 2007; Hammet et al. 2007). Phosphorylated Rad9 induces checkpoint Rad53 and Chk1 activation, resulting in several responses, including cell cycle arrest (Weinert and Hartwell 1993).

Evidences for checkpoint protein involvement in chromatin motion come from studies where the effect of mec1, rad9 and rad53 deletions on chromatin mobility was studied ${ }^{2}$. Chromatin mobility was examined through measurement of $R_{c}$ at long time points ( 5 minutes) by tracking a genomic locus or alternatively, a Rad52-GFP focus taken as a marker of genomic break (Dion et al. 2012; Seeber et al. 2013). In all mutants, $R_{c}$ did not increase upon Zeocin treatment (Lisby et al. 2001; Dion et al. 2012; Seeber et al. 2013). Consequently, a role for Rad9 induced motion in homologous recombination was tested in G2/M arrested cells (arrest is used to avoid subsequent cell cycling due to Rad9 deletion), full repair by homologous recombination was found decreased in $\Delta$ rad9, implicating Rad9 in long range homology search (Dion et al. 2012).

2. Histone modifications. It is not surprising that mutations of structural chromatin components can cause motion changes at the site of damage. Effects elsewhere in the genome are less intuitive. In our recent study, we showed that genomic damage by Zeocin resulted in increased intra-chromosomal distances concomitant to increased chromatin subdiffusion, an observation that polymer modeling could explain by chromatin stiffening (Herbert et al. 2017). This stiffening was further supported by super-resolution STORM/PALM microscopy observations (Herbert et al. 2017). One possible explanation for stiffening could be the negative charges due to H2A S129

\footnotetext{
${ }^{2}$ Study of $\Delta m e c 1$ or $\Delta r a d 53$ is hampered by the fact that these genes are essential, unless Sml1 is also deleted. The increased uptake of dNTPs in this mutated $\Delta s m / 1$ background renders cells less sensitive to the lack of Mec1 or Rad53
} 
phosphorylation, since the Zeocin dependent increase in intrachromosomal distances was reduced H2A S129A mutant (Herbert et al. 2017). Interestingly, a concomitant study analyzed chromatin behavior by multi-scale tracking after exposure to $\gamma$-rays, leading to $\sim 4$ random DSBS across the genome, or after an I-Scel induced DSB (Miné-Hattab et al. 2017). MSD was reduced at small time scales but MSD increased at longer time scales (Miné-Hattab et al. 2017). These different patterns of chromatin mobility are consistent with a global increase in chromatin stiffening (Faller and Müller-Plathe 2001). Intriguingly, in the presence of Zeocin, an impressive, nhp6 dependent, degradation of histones $\mathrm{H} 3$ and $\mathrm{H} 4$ (up to $20-40 \%$ ) is observed (Hauer et al. 2017). Whereas a different polymer modeling interprets chromatin structural changes as chromatin decompaction, rather than stiffening (Amitai et al. 2017), chromatin MSD is shown to similarly increase with an $\alpha$ value rising from 0.5 to up to 0.7. Although the changes in the physical parameters of compaction and/or stiffening are not yet fully understood, these concomitant studies all point to the important global chromatin structural alterations challenged by DNA damages (Miné-Hattab et al. 2017; Hauer et al. 2017; Herbert et al. 2017).

2. Repair proteins Factors involved in the repair process itself (sae2, rad54, rad52) are shown to be involved in the pronounced changes of local motion of damaged chromatin, but no clear role has been established in global chromatin dynamics, with the exception of Rad51 (Miné-Hattab and Rothstein 2012; Miné-Hattab et al. 2017). Upon zeocin treatment or $\gamma$ ray irradiations, global mobility is increased in a Rad51 dependent manner through a mechanism that remains to be elucidated (Seeber et al. 2013; Miné-Hattab et al. 2017) in agreement with observations made in drosophila

3. Chromatin remodelers and cytoskeleton proteins. It was shown that Arp8 is at least in part involved in enhanced mobility of damaged chromatin, since mobility of a genomic locus (2.8kb from I-Scel cutting site) is decreased at long time points in a $\Delta$ arp8 strain (Neumann et al. 2012). Arp8 and Arp5, both members of the INO80 complex are also involved in DSB induced global mobility (Seeber et al. 2013). Intriguingly, a role for nuclear microtubules in chromatin mobility upon I-Scel cleavage has been recently shown for an interstitial locus far from tethering structures (Lawrimore et al. 2017). It is not known how microtubules act on damaged chromatin. Perhaps nuclear dynein is important in this process, as suggested (Chung et al. 2015).

Altogether these studies show that global mobility, observed in trans upon DNA damage, is regulated by multiple factors whose interplay has to be deciphered. Understanding how transmobility is established remains an exciting and important challenge.

B. Chromosome organization and tethering features of the nucleus. 
Combination of high-throughput imaging of genomic loci in individual cells, chromosome capture techniques and chromosome modeling based on polymer physics has established that yeast chromosomes follow a Rabl-like configuration (Jin et al. 2000; Schober et al. 2008; Therizols et al. 2010; Duan et al. 2010; Cournac et al. 2012; Wong et al. 2012; Arbona et al. 2017). In this configuration, anchoring to nuclear microtubules tethers centromeres close to the Spindle Pole Body - which in yeast is embedded in the nuclear envelope - and telomeres are found close to the nuclear envelope in a position dependent on the chromosome arm size. By searching for factors phosphorylated by Mec1 upon DNA damage, Strecker et al found Cep3, a kinetochore protein (Strecker et al. 2016). If Cep3 is mutated for its phosphorylation site (S175A), increase of $\mathrm{R}_{\mathrm{c}}$ generated by $\mathrm{HO}$ endonuclease is inhibited, for loci both in cis and trans of the break (Strecker et al. 2016). It is proposed that Cep3 phosphorylation by Mec1 mediates chromatin mobility through relaxation of the kinetochore-centromere link (Strecker et al. 2016). The nature of this relaxation remains to be further comprehended. Lawrimore et al., observed an expanded chromatin at centromeres and implicated microtubules in the enhanced movement of damaged chromatin (Lawrimore et al. 2017). Zeocin treatment also led to similar increase in distances between SPB and a centromere proximal loci is found in WT cells strains in WT cells and cep3 mutated cells (Herbert et al. 2017). These observations are complicated by the concomitant G2/M checkpoint arrest. Of note, another checkpoint, the spindle assembly checkpoint (SAC), prevents aberrant chromosome segregation and aneuploidy. SAC controls chromosome separation through inactivation of the Securin/Pds1 (George and Walworth 2016). Pds1 is known to inhibit the separase /Esp1 itself involved in the cleavage of the cohesin that holds sister chromatids after replication. Cleavage by Esp1 is required for sister chromatid segregation (Uhlmann et al. 1999). It has been recently shown that SAC is active even in the absence of Mec1 and Tel1 (Palou et al. 2016). Analysis of chromatin mobility in this mutated context should certainly clarify how G2/M checkpoint controls chromosome mobility. Tethering of telomeres might also be modified upon DSB, since deletions of SIR4, or KU70, both proteins involved in telomere anchoring at the nuclear periphery, mirror the mobility increase observed upon HO cleavage (Strecker:2016jj; Lawrimore et al. 2017). One explanation for this observation could come from work showing that upon DSB, Ku70 relocates from telomeres to DSB ends (Martin et al. 1999; Walker et al. 2001), but mutants for which telomere tethering would not be modified upon DNA damage are not characterized to date.

\section{Functional roles for chromatin mobility?}


What could be the functional role for increased chromatin mobility? In yeast, several studies have shown that enhanced DNA mobility positively correlates with repair efficiency, as judged by transformation frequencies or molecular recombination events upon a DSB, as compared to the absence of DSB. For instance, targeting Arp8 or Ino80, increases the rate of homologous recombination. On the contrary, delayed motion due to latrunculin or mutation in the catalytic subunit of INO80 complex, decreases homologous recombination rates (Neumann et al. 2012; Spichal et al. 2016). Defect in mobility due to rad9 deletion also delays the appearance of recombination intermediates (Dion et al. 2012). A limitation in these studies remains in the difficulty to find mutants where mobility function is distinct from the repair function. In this regard, cep3 mutant, that limits chromatin motion and is not a repair protein per se, had no impact on recombination frequency (Strecker et al. 2016). The role of chromatin motion in repair by homologous recombination therefore remains an open question. It is however consistent to find a correlation between increased subdiffusion and increased repair efficiency, since it could ensure a greater probability of encounter between broken molecules. But because the temporal windows are difficult to reconcile, since mobility can be observed minutes after breakage, and repair events are followed days after it, it leaves open the possibility that increased mobility does not directly impact homology search and repair. Another possibility could be that an increased mobility helps moving the damaged chromatin towards nuclear compartments prone to repair (Therizols et al. 2006; Nagai et al. 2008). Conversely, it cannot be excluded that, as proposed for telomeres mobility in mammals, increased mobility could favour unwanted translocations, if not properly regulated (Cho et al. 2014; Lottersberger et al. 2015). Could it also be that increased motion is an inherent response of cells to DNA damage, with no obvious function in repair? Understanding the how and why of chromatin mobility upon double strand breaks thus still awaits fascinating studies.

\section{Acknowledgements}

This review is dedicated to Cécile Fabre Martial. We thank Karine Dubrana, Judith Miné-Hattab, JeanMarc Victor for constructive comments about our manuscript, Etienne Almayrac and Fabiola Garcia Fernandez for their contribution to the figures and members of the consortium GDR ADN (Architecture du Noyau) for lively discussions. C.Z. acknowledges funding by Institut Pasteur, Institut National du Cancer (INCa 2015-135), Fondation pour la Recherche Médicale (Equipe FRM DEQ20150331762). E.F. acknowledges support from Agence Nationale de la Recherche (ANR-13BSV8-0013-01), IDEX SLI (DXCAIUHSLI-EF14), Labex Who am I (ANR-11-LABX-0071, Idex ANR-11-IDEX0005-02), Cancéropôle Ile de France (ORFOCRISE PME-2015) and Fondation pour la Recherche Médicale (ING20160435205). 
Figure 1 Molecular scenario at the site of a DSB

Involvement of checkpoint, histone $\mathrm{H} 2 \mathrm{~A}$ phosphorylation, remodeling complexes, and repair proteins at the site of DNA damage are depicted.

Figure 2: Possible mechanisms for chromatin mobility upon DNA damage.

Four chromosomes in a Rabl configuration of a haploid yeast cell are shown (A). In undamaged conditions, chromosomes are tethered to the spindle pole body (SPB) by their centromere (CEN) via a nuclear microtubule (MT) and to the nuclear envelope (NEV) by their telomere (TEL). (B) Possible scenarios of enhanced chromatin dynamics following DNA damage at the site of DNA damage is shown. Proposed mechanism for global mobility include (C) stiffening (or alternatively, decondensation) of the chromatin fiber and (D) relaxation of the link between centromere and SPB through microtubule, loss of telomeres tethering and impaired function of nuclear actin.

Table 1 Effects of mutants in cis (local) or trans mobility (global) are recapitulated. Increase in motion upon DNA damage is indicated as "inhibited ", when it is similar to the WT motion without DNA damage. Effects upon Zeocin treatment are also shown. Note that all these studies follow locus motion for $\Delta t$ around 180 seconds after 3hours of DNA damage induction. Studies in haploid and diploid cells are distinguished, since ploidy has an effect in chromatin mobility. Local mobility refers to the motion of a locus close to an enzymatic cutting site. Trans mobility can refer to the motion of a locus on a chromosome distinct from the chromosome carrying the enzymatic cutting site or to the motion due to a genotoxic random agent like Zeocin.

\section{References}

Albert B, Léger-Silvestre I, Normand C, Gadal O (2012) Nuclear organization and chromatin dynamics in yeast: Biophysical models or biologically driven interactions? BBA - Gene Regulatory Mechanisms 1-14. doi: 10.1016/j.bbagrm.2011.12.010

Amitai A, Seeber A, Gasser SM, Holcman D (2017) Visualization of Chromatin Decompaction and Break Site Extrusion as Predicted by Statistical Polymer Modeling of Single-Locus Trajectories. CellReports 18:12001214. doi: 10.1016/j.celrep.2017.01.018

Arbona J-M, Herbert S, Fabre E, Zimmer C (2017) Inferring the physical properties of yeast chromatin through Bayesian analysis of whole nucleus simulations. Genome Biol 18:81. doi: 10.1186/s13059-017-1199-x

Backlund MP, Joyner R, Weis K, Moerner WE (2014) Correlations of three-dimensional motion of chromosomal loci in yeast revealed by the double-helix point spread function microscope. Mol Biol Cell 25:3619-3629. doi: 10.1091/mbc.E14-06-1127

Barlow JH, Lisby M, Rothstein R (2008) Differential regulation of the cellular response to DNA double-strand breaks in G1. Mol Cell 30:73-85. doi: 10.1016/j.molcel.2008.01.016 
Bonilla CY, Melo JA, Toczyski DP (2008) Colocalization of sensors is sufficient to activate the DNA damage checkpoint in the absence of damage. Mol Cell 30:267-276. doi: 10.1016/j.molcel.2008.03.023

Bronshtein I, Kepten E, Kanter I, et al (2015) Loss of lamin A function increases chromatin dynamics in the nuclear interior. Nature Communications 6:1-9. doi: 10.1038/ncomms9044

Cabal GG, Genovesio A, Rodriguez-Navarro S, et al (2006) SAGA interacting factors confine sub-diffusion of transcribed genes to the nuclear envelope. Nature 441:770-773. doi: 10.1038/nature04752

Cho NW, Dilley RL, Lampson MA, Greenberg RA (2014) Interchromosomal Homology Searches Drive Directional ALT Telomere Movement and Synapsis. cell 159:108-121. doi: 10.1016/j.cell.2014.08.030

Chubb JR, Boyle S, Perry P, Bickmore WA (2002) Chromatin motion is constrained by association with nuclear compartments in human cells. Curr Biol 12:439-445.

Chung DKC, Chan JNY, Strecker J, et al (2015) Perinuclear tethers license telomeric DSBs for a broad kinesinand NPC-dependent DNA repair process. Nature Communications 6:1-13. doi: 10.1038/ncomms8742

Cournac A, Marie-Nelly H, Marbouty M, et al (2012) Normalization of a chromosomal contact map. BMC Genomics 13:436. doi: 10.1186/1471-2164-13-436

Dion V, Kalck V, Horigome C, et al (2012) Increased mobility of double-strand breaks requires Mec1, Rad9 and the homologous recombination machinery. Nat Cell Biol 14:502-509. doi: 10.1038/ncb2465

Duan Z, Andronescu M, Schutz K, et al (2010) A three-dimensional model of the yeast genome. Nature. doi: 10.1038/nature08973

Ehrenfeld GM, Shipley JB, Heimbrook DC, et al (1987) Copper-dependent cleavage of DNA by bleomycin. Biochemistry 26:931-942.

Faller R, Müller-Plathe F (2001) Chain stiffness intensifies the reptation characteristics of polymer dynamics in the melt. Chemphyschem 2:180-184. doi: 10.1002/1439-7641(20010316)2:3<180::AID-

CPHC180>3.0.CO;2-Z

Finn K, Lowndes NF, Grenon M (2011) Eukaryotic DNA damage checkpoint activation in response to doublestrand breaks. Cell Mol Life Sci 69:1447-1473. doi: 10.1007/s00018-011-0875-3

George AA, Walworth NC (2016) Microtubule dynamics decoded by the epigenetic state of centromeric chromatin. Curr Genet 62:691-695. doi: 10.1007/s00294-016-0588-0

Gibb B, Ye LF, Kwon Y, et al (2014) Protein dynamics during presynaptic-complex assembly on individual single-stranded DNA molecules. Nat Struct Mol Biol 21:893-900. doi: 10.1038/nsmb.2886

Grenon M, Costelloe T, Jimeno S, et al (2007) Docking onto chromatin via the Saccharomyces cerevisiae Rad9 Tudor domain. Yeast 24:105-119. doi: 10.1002/yea.1441

Hajjoul H, Mathon J, Ranchon H, et al (2013) High-throughput chromatin motion tracking in living yeast reveals the flexibility of the fiber throughout the genome. Genome Res 23:1829-1838. doi: 10.1101/gr.157008.113

Hammet A, Magill C, Heierhorst J, Jackson SP (2007) Rad9 BRCT domain interaction with phosphorylated H2AX regulates the G1 checkpoint in budding yeast. EMBO Rep 8:851-857. doi: 10.1038/sj.embor.7401036

Hauer MH, Seeber A, Singh V, et al (2017) Histone degradation in response to DNA damage enhances chromatin dynamics and recombination rates. Nat Struct Mol Biol 1-13. doi: 10.1038/nsmb.3347

Herbert S, Brion A, Arbona J-M, et al (2017) Chromatin stiffening underlies enhanced locus mobility after DNA 
damage in budding yeast. EMBO J 36:2595-2608. doi: 10.15252/embj.201695842

Heun P (2001) Chromosome Dynamics in the Yeast Interphase Nucleus. 294:2181-2186. doi:

10.1126/science.1065366

Jin QW, Fuchs J, Loidl J (2000) Centromere clustering is a major determinant of yeast interphase nuclear organization. J Cell Sci 113 ( Pt 11):1903-1912.

Lawrimore J, Barry TM, Barry RM, et al (2017) Microtubule dynamics drive enhanced chromatin motion and mobilize telomeres in response to DNA damage. Mol Biol Cell 28:1701-1711. doi: 10.1091/mbc.E16-120846

Lee C-S, Lee K, Legube G, Haber JE (2014) SI Dynamics of yeast histone H2A and H2B phosphorylation in response to a double-strand break. Nat Struct Mol Biol 21:103-109. doi: 10.1038/nsmb.2737

Lisby M, Rothstein R, Mortensen UH (2001) Rad52 forms DNA repair and recombination centers during S phase. Proc Natl Acad Sci USA 98:8276-8282. doi: 10.1073/pnas.121006298

Lottersberger F, Karssemeijer RA, Dimitrova N, de Lange T (2015) 53BP1 and the LINC Complex Promote Microtubule- Dependent DSB Mobility and DNA Repair. cell 163:880-893. doi: 10.1016/j.cell.2015.09.057

Marshall WF, Straight A, Marko JF, et al (1997) Interphase chromosomes undergo constrained diffusional motion in living cells. Curr Biol 7:930-939.

Martin SG, Laroche T, Suka N, et al (1999) Relocalization of telomeric Ku and SIR proteins in response to DNA strand breaks in yeast. cell 97:621-633.

Miné-Hattab J, Recamier V, Izeddin I, Rothstein R \& Darzacq X (2017) Multi-scale tracking reveals scale-dependent chromatin dynamics after DNA damage. Mol Biol Cell 28: 3323-3332

Miné-Hattab J, Rothstein R (2012) Increased chromosome mobility facilitates homology search during recombination. Nat Cell Biol 1-12. doi: 10.1038/ncb2472

Nagai S, Dubrana K, Tsai-Pflugfelder M, et al (2008) Functional Targeting of DNA Damage to a Nuclear PoreAssociated SUMO-Dependent Ubiquitin Ligase. 322:597. doi: 10.1126/science.1162790

Neumann FR, Dion V, Gehlen LR, et al (2012) Targeted INO80 enhances subnuclear chromatin movement and ectopic homologous recombination. Genes Dev 26:369-383. doi: 10.1101/gad.176156.111

Palou R, Palou G, Quintana DG (2016) A role for the spindle assembly checkpoint in the DNA damage response. Curr Genet 63:275-280. doi: 10.1007/s00294-016-0634-y

Saad H, Gallardo F, Dalvai M, et al (2014) DNA Dynamics during Early Double-Strand Break Processing Revealed by Non-Intrusive Imaging of Living Cells. PLoS Genet 10:e1004187-11. doi: 10.1371/journal.pgen.1004187

Schober H, Kalck V, Vega-Palas MA, et al (2008) Controlled exchange of chromosomal arms reveals principles driving telomere interactions in yeast. Genome Res 18:261-271. doi: 10.1101/gr.6687808

Seeber A, Dion V, Gasser SM (2013) Checkpoint kinases and the INO80 nucleosome remodeling complex enhance global chromatin mobility in response to DNA damage. Genes Dev 27:1999-2008. doi: 10.1101/gad.222992.113

Shroff R, Arbel-Eden A, Pilch D, et al (2004) Distribution and Dynamics of Chromatin Modification Induced by a Defined DNA Double-Strand Break. Current Biology 14:1703-1711. doi: 10.1016/j.cub.2004.09.047

Spichal M, Brion A, Herbert S, et al (2016) Evidence for a dual role of actin in regulating chromosome 
organization and dynamics in yeast. J Cell Sci 129:681-692. doi: 10.1242/jcs.175745

Spichal M, Fabre E (2017) The Emerging Role of the Cytoskeleton in Chromosome Dynamics. Front Genet 8:587-12. doi: 10.3389/fgene.2017.00060

Steighner RJ, Povirk LF (1990) Bleomycin-induced DNA lesions at mutational hot spots: implications for the mechanism of double-strand cleavage.

Steinhauser MO, Schneider J, Blumen A (2009) Simulating dynamic crossover behavior of semiflexible linear polymers in solution and in the melt.

Strecker J, Gupta GD, Zhang W, et al (2016) DNA damage signalling targets the kinetochore to promote chromatin mobility. Nat Cell Biol 18:281-290. doi: 10.1038/ncb3308

Tam ATY, Pike BL, Hammet A, Heierhorst J (2007) Telomere-related functions of yeast KU in the repair of bleomycin-induced DNA damage. Biochem Biophys Res Commun 357:800-803. doi:

10.1016/j.bbrc.2007.04.011

Therizols P, Duong T, Dujon B, et al (2010) Chromosome arm length and nuclear constraints determine the dynamic relationship of yeast subtelomeres. Proceedings of the National Academy of Sciences 107:20252030. doi: $10.1073 /$ pnas.0914187107

Therizols P, Fairhead C, Cabal GG, et al (2006) Telomere tethering at the nuclear periphery is essential for efficient DNA double strand break repair in subtelomeric region. J Cell Biol 172:189-199. doi: 10.1083/jcb.200505159

Tsukuda T, Fleming AB, Nickoloff JA, Osley MA (2005) Chromatin remodelling at a DNA double-strand break site in Saccharomyces cerevisiae. Nature 438:379-383. doi: 10.1038/nature04148

Uhlmann F, Lottspeich F, Nasmyth K (1999) Sister-chromatid separation at anaphase onset is promoted by cleavage of the cohesin subunit Scc1. Nature 400:37-42. doi: 10.1038/21831

Verdaasdonk JS, Vasquez PA, Barry RM, et al (2013) Centromere Tethering Confines Chromosome Domains. Mol Cell 52:819-831. doi: 10.1016/j.molcel.2013.10.021

Walker JR, Corpina RA, Goldberg J (2001) Structure of the Ku heterodimer bound to DNA and its implications for double-strand break repair. Nature 412:607-614. doi: 10.1038/35088000

Weber SC, Spakowitz AJ, Theriot JA (2010) Bacterial chromosomal loci move subdiffusively through a viscoelastic cytoplasm. Phys Rev Lett 104:238102. doi: 10.1103/PhysRevLett.104.238102

Weinert TA, Hartwell LH (1993) Cell cycle arrest of cdc mutants and specificity of the RAD9 checkpoint. Genetics 134:63-80.

Wong H, Marie-Nelly H, Herbert S, et al (2012) A predictive computational model of the dynamic 3D interphase yeast nucleus. Curr Biol 22:1881-1890. doi: 10.1016/j.cub.2012.07.069

Zhu Z, Chung W-H, Shim EY, et al (2008) Sgs1 helicase and two nucleases Dna2 and Exo1 resect DNA doublestrand break ends. cell 134:981-994. doi: 10.1016/j.cell.2008.08.037 